\title{
Dynamical properties of long-wavelength interface fluctuations during nucleation-dominated crystal growth
}

\author{
Wim van Saarloos and George H. Gilmer \\ AT\&T Bell Laboratories, Murray Hill, New Jersey 07974
}

(Received 25 October 1985)

\begin{abstract}
Motivated by advances in laser scattering experiments on crystal-melt interfaces during growth conditions, we discuss the interface fluctuations of a faceted crystal during nucleation-dominated growth. If the nucleation rate is large enough so that each layer grows as a result of multiple nucleation events, the properties of long-wavelength interface fluctuations can be understood in terms of a simple random-walk picture. Accordingly, apart from a change in length scale, the interface fluctuations during growth resemble those of a rough interface. This is a form of dynamic roughening. The various factors contributing to the decay of fluctuations are discussed and a comparison with a model of Bilgram et al. for the interface dynamics is made.
\end{abstract}

\section{INTRODUCTION}

In this paper we will discuss the long-wavelength interface fluctuations that occur when a crystal grows via the nucleation of new layers. As a simple model for nucleation-dominated growth, we will study the polynuclear growth (PNG) model. ${ }^{1,2}$ The PNG model consists of disk-shaped clusters that nucleate at random positions and then expand until they merge with other clusters in the same layer, thus forming a multilevel structure of the type sketched in Fig. 1. We will show that the longwavelength interface fluctuations can be understood in terms of a simple random-walk picture, and that, as a result, the long-wavelength properties of the model resemble those of a "rough" interface." 3 The model therefore illustrates the idea of "dynamic roughening;" i.e., the fact that when viewed on the proper length scale, even the interface of a faceted crystal becomes rough during growth conditions.

The work described in this paper was motivated in part by recent light scattering experiments, ${ }^{5-12}$ by which the microscopic growth mechanism of a crystal is investigated through the study of interface fluctuations. To put these experiments into proper perspective, we first summarize the various growth mechanisms and the difficulty of probing them experimentally. ${ }^{13}$

When a crystal-melt or crystal-vapor interface is microscopically "rough", the equilibrium shape of a crystal is rounded, and the growth rate $R$ of the interface is linear in the driving force $\Delta T=T_{m}-T_{i}$, the difference between the equilibrium melting temperature $T_{m}$ and the interface temperature $T_{i}$. (See, e.g., Refs. 3 and 4 for general reviews of crystal growth.) For a faceted interface, on the other hand, the free-energy $\sigma$ associated with the formation of a step is finite. A faceted interface therefore grows via the nucleation and spreading of new layers or by means of growth spirals emanating from screw dislocations that provide a continuous source of steps on the surface. In the first case of nucleation dominated growth, the nucleation barrier is of the order of $\pi \sigma^{2} / \Delta \mu$ and so the nucleation rate is proportional to $\exp \left[-\pi \sigma^{2} /(k T \Delta \mu)\right]$. (Here $\Delta \mu$ is the difference in chemical potential between the crystal and melt or vapor.) Hence for $T_{i}$ close enough to $T_{m}$ the nucleation probability is extremely small (since $\Delta \mu \rightarrow 0$ for $T_{i} \rightarrow T_{m}$ ), and the growth usually becomes dominated by the spiral mechanism resulting from the steps already present at positions where screw dislocations intersect the interface. The growth rate due to the latter mechanism alone is proportional to $(\Delta T)^{2}$.

With the aid of computer simulation, it has been possible to investigate each of the above mechanisms separately, e.g., by considering systems with and without dislocations, or by suppressing nucleation, etc. ${ }^{3}$ Such studies have verified the essential correctness of the above picture in detail. Experimentally, it is of course less easy to isolate the various contributions. In a typical experiment the growth rate of the crystal $R$ is measured as a function of undercooling $\Delta T$. The growth mechanism is then inferred from a comparison of the experimental data with the functional $R$ versus $\Delta T$ dependence associated with the various mechanisms discussed above. For a number of faceted materials, it has been possible to do precise experiments on crystals without screw dislocations, and the experimental observations do indeed show several characteristics of nucleation type growth. ${ }^{14}$ However, since the expressions for growth controlled by nucleation events in-

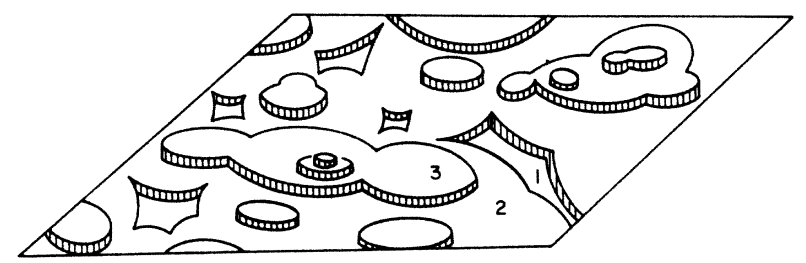

FIG. 1. Schematic view of an interface in the PNG model. Layer 1 has been fully completed, while the clusters in layer 2 are about to fill the last holes by merging. In the next layer, 3, already several clusters have nucleated and some of them are merging. A few small clusters have nucleated in the next layer too. 
volve several quantities whose values are not well known, it is generally difficult from these experiments to extract accurate quantitative information on important surface quantities like the step free energy, the step velocity, etc. Moreover, in less controlled experiments of this kind, the growth of a faceted interface may be due to both nucleation and dislocations. In such cases, the growth-rate data are usually fitted by a $R \sim(\Delta T)^{\gamma}$ law over a certain range of temperatures, with the effective exponent $\gamma$ depending on the material, the temperature range, etc. Obviously, it can often be difficult to decide whether data of this form should be interpreted as resulting from the combined effect of the two growth mechanisms, or as a signature of the presence of other important phenomena.

The above discussion illustrates the desirability of having independent experimental techniques to probe surface quantities of crystals during growth conditions. Laser light scattering experiments of the kind initiated by Bilgram and co-workers ${ }^{5}$ in 1978 have been shown to be a powerful tool in this respect. ${ }^{15}$ Bilgram et al. ${ }^{5-10}$ and later also Mesquita et al. ${ }^{11}$ and Brown et al. ${ }^{12}$ have studied the interfacial correlations of ice and salol during growth conditions. Although, as will be discussed later, the origin of the so-called diffusive scattering seen in these experiments is not understood at present, clear evidence for the existence of growth spirals was obtained in the studies of salol. ${ }^{8,10,11}$ The light scattering observed under condition of total reflection within the crystal ${ }^{11}$ was highly anisotropic and had an oscillatory time correlation function. This was interpreted as the light scattered off polygonized growth spirals emanating from screw dislocations. The oscillatory correlation of the scattered light is then caused by the Doppler shift resulting from the propagation of steps with velocity $V_{s}$. These experiments therefore do not only probe the growth mechanism of the crystal, but also yield direct in situ measurements of $V_{s}$ as well.

We discuss in this paper the long-wavelength interface correlation of a crystal-melt or crystal-vapor interface during nucleation-dominated growth. Although the average growth rate as a function of nucleation rate and step velocity in this regime has been studied extensively, the dynamics of interface fluctuations have, to our knowledge, not been discussed before. One of the main goals of this paper is to show that these dynamics can be understood quite simply in terms of a random-walk picture. As a result of this, the interface correlations during nucleation-dominated growth strongly resemble those of a rough interface. In other words, an interface that is faceted at equilibrium "dynamically roughens" on long length scales as soon as it is forced to grow. When a faceted interface is far below the equilibrium roughening temperature, this dynamically induced roughness at small driving force (nucleation rate) will only affect the interface properties at very long length scales. Thus, two interfaces can still be very different at shorter length scales when they have similar correlations in height at large distance scales. The interface morphology at shorter length scales will not be considered in this paper, however, as it cannot simply be characterized in terms of surface roughening, a concept which can only be defined properly as the divergence of the interface width in the long-wavelength limit. ${ }^{16}$

In Sec. II, we introduce the PNG model and qualitatively discuss the long-wavelength interface fluctuations in this model. We then present (Sec. III) the data of numerical simulations that support the qualitative picture of Sec. II and the idea of dynamic roughening. In Sec. IV the model of interface fluctuations of Bilgram et al. ${ }^{5,6}$ is summarized and the underlying physics is compared with that of the random PNG model. The dynamics of fluctuations in the PNG model is of purely kinematic origin (the spreading and merging of clusters), while the decay of fluctuations in Bilgram's model is related to the dependence of the growth rate on the local structure of the interface. In general, both effects will be present, but for small growth rates, we expect the first effect to dominate. In Sec. $\mathrm{V}$ we discuss the experimental implications of our results. Several of the arguments presented for the PNG model in a two-dimensional interface can be illustrated by explicit calculations for the PNG model on a onedimensional interface. This is done in Sec. VI, where we allow the nucleation rate of the one-dimensional PNG model to depend on the curvature of the interface. In this case the dynamics of fluctuations can be obtained exactly; the long-wavelength diffusive behavior indeed exhibits the combined effect of the spreading and merging of clusters and of the curvature dependence of the growth rate considered by Bilgram et al.

\section{RANDOM-WALK PICTURE OF FLUCTUATIONS IN THE PNG MODEL}

We consider here the nucleation controlled growth of an infinite crystal surface. In the PNG model, 1,2 clusters nucleate at random sites on the surface of the crystal at a rate $J$ per unit area. The clusters start with zero radius at the time of nucleation and expand with radial speed $V$ that is independent of the radius. Each piece of the edge of a cluster continues growing until it merges with the edge of another cluster in the same layer. A layer is completed when all clusters in that layer have merged. Figure 1 gives an idea of the resulting surface.

The above model is based on a somewhat idealized description of nucleation events. In reality, clusters nucleate with a finite radius $\rho_{c}$ and their growth velocity is a function of the radius. In the above model, $\rho_{c}(=0)$ is underestimated but $V$ is overestimated for small radii, so the effects of the two approximations partly cancel. In any case, these approximations will be justified if $\rho_{c}$ is much smaller than the typical size a cluster reaches before merging. Furthermore, since we consider disk-shaped clusters, we neglect possible anisotropic growth kinetics of steps. Such anisotropies will not qualitatively affect the longwavelength interface fluctuations, however. The most important simplification in the model is that $J$ is a fixed constant, independent of time and of the local surface structure. In reality, the nucleation rate may depend on the interface structure, e.g., on the height of the surface through the temperature or supersaturation. These effects will be discussed later in Sec. IV; we will first focus on the dynamics resulting from completely random nucleation events, and refer to this random model as the PNG model. 
Gilmer $^{2}$ has studied the initial growth transients of the PNG model and also compared the data with Ising model Monte Carlo simulations to assess the importance of atomistic effects. ${ }^{17}$ Here we will only discuss steady-state interface fluctuations.

In the PNG model, there are only two parameters, $J$ and $V$. Their dimensions, indicated by [], are

$$
[J]=1 /\left(\text { length }{ }^{2} \text { time }\right),[V]=\text { length } / \text { time . }
$$

These parameters define a length scale $\lambda$ and a time scale $\tau$ as

$$
\lambda \equiv\left(\frac{V}{J}\right)^{1 / 3}, \tau \equiv\left(J V^{2}\right)^{-1 / 3} .
$$

The simplicity of the PNG model lies in the fact that it has no free parameters that affect the qualitative behavior; $J$ and $V$, and hence $\lambda$ and $\tau$, only set the proper scales. For instance, the growth rate $R$ and the number of layers the crystal grows per unit of time must, on dimensional grounds, be of the form

$$
R=\frac{c_{1}}{\tau},
$$

with $c_{1}$ a dimensionless constant. From the numerical simulations of Gilmer, ${ }^{2}$ it is found that $c_{1} \simeq 1.40$. $^{18}$ This value indicates that the typical size a cluster reaches before merging with the others if of order $\lambda$. To see this, note that the $J$ clusters that land in a unit area per unit of time grow $R$ layers. $J / R$ clusters cover one unit area in one layer, so the effective area covered by one cluster is $R / J$. This suggests that we can define the effective cluster radius $r$ in terms of the average area a cluster covers as

$$
\pi r^{2} \equiv \frac{R}{J}
$$

Using (1) and (2), this yields

$$
r=\left(\frac{c_{1}}{\pi \tau J}\right)^{1 / 2}=\left(\frac{c_{1}}{\pi}\right)^{1 / 2} \lambda \simeq 0.67 \lambda .
$$

Similarly, the typical "lifetime" of a cluster is $r / V \simeq 0.67 \tau$.

Let us now discuss fluctuations of the interface in a steady state. Consider an initial long-wavelength fluctuation of the interface with larger than average height as indicated in Fig. 2(a). Since we are interested in the dynamics of fluctuations about the average height of the interface, it is important to note that the dynamics of interface fluctuations should be measured in a coordinate system that moves with the average interface position as the crystal grows; in this system, the average height remains at a fixed position. The time evolution of a fluctuation is governed by two processes, the spreading of clusters and the nucleation of new layers on top of them. In every layer, a cluster has a finite lifetime of the order of $\tau$, so that during the first time interval of order $\tau$, the height fluctuation spreads out laterally over a distance of order $r \simeq \lambda$. At the same time, new clusters nucleate at random positions on top of the old clusters that formed the initial perturbation. Since their probability of formation during

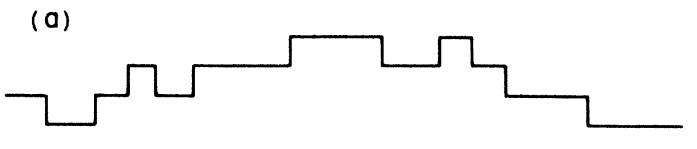

(b)

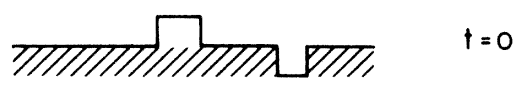

(c)

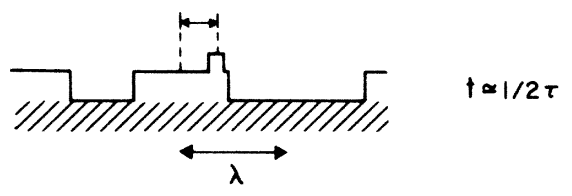

(d)

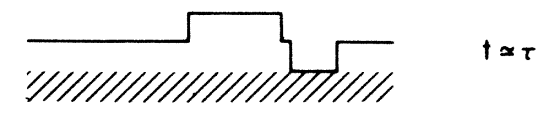

FIG. 2. (a) Schematic view of a long-wavelength height fluctuation in a one-dimensional PNG model. (b)-(d) Illustration of the random-walk nature of the propagation of height fluctuations for the idealized situation of a single cluster. (b) Initial condition with a small cluster in a new layer. (c) After a time of order $\frac{1}{2} \tau$, other clusters have nucleated in the same layer as the cluster in (b). Moreover, a new one has nucleated on top of the old one. (d) After a time of order $\tau$, the crystal has grown one layer, and the interface resembles the one in (b). However, the center of the top cluster in (d) is a distance of order $\frac{1}{2} \lambda$ to the right of the original one.

the initial period was enhanced at the position of the original fluctuation, the influence of the height fluctuation persists to the next layer. However, the center of the new cluster will generally be displaced somewhat with respect to the position of the center of the cluster below, because of the random nature of the nucleation events. This is illustrated in Figs. 2(b)-2(d) for a one-dimensional interface. Clearly, although on the average the center of the new cluster will be centered above the old one, it will typically be displaced by a distance of order $\lambda$ in a random direction. Therefore, the time evolution of average height fluctuations corresponds to the lateral diffusion process of a random walker that makes steps of order $r \simeq \lambda$ in a time $\tau$. We therefore expect for the spatial Fourier transform of $h(\mathbf{r}, t)$, the deviation from the average height of the interface,

$$
\left\langle h(\mathbf{k}, t) h^{*}(\mathbf{k}, 0)\right\rangle \sim\left\langle h(\mathbf{k}, 0) h^{*}(\mathbf{k}, 0)\right\rangle e^{-D_{\mathrm{kin}} k^{2} t},
$$

where $D_{\text {kin }}$ is the diffusion coefficient associated with the kinematics of the spreading and merging of clusters. The random-walk picture suggests that

$$
D_{\mathrm{kin}} \simeq \frac{\lambda^{2}}{\tau}
$$

In (5),

$$
h(\mathbf{k}, t)=\int d \mathbf{r} e^{i \mathbf{k} \cdot \mathbf{r}} h(\mathbf{r}, t)
$$


is a lateral Fourier transform, \langle\rangle stands for an ensemble average, and * denotes the complex conjugate. In Sec. III, we show that numerical simulations of the PNG model support the above picture, and the validity of Eqs. (5) and (6). The above discussion indicates that during nucleation dominated growth the long-wavelength structure of the interface is determined by the presence of steps. The surface structure therefore resembles that of a rough interface, i.e., it has "dynamically roughened." However, one should keep in mind that this dynamic roughening only occurs on long length scales $l \gg \lambda_{;}^{19}$ as explained in the Introduction, the interface properties at shorter length scales depend on other factors such as the closeness of the equilibrium roughening transition.

In an experiment with scattering vector $\mathbf{k}$, the correlation of interface fluctuations with wave vector $\mathbf{k}_{\|}$, the component of $\mathbf{k}$ parallel to the interface, is probed. Like the corrugated interface model of Bilgram et al. ${ }^{5,6}$ to be discussed later, correlations are damped with $k_{\|}^{2}$ rather than $k^{2}$. This anisotropy can be made somewhat more precise by further exploiting the resemblance of the interface structure to that of a rough interface. The longwavelength excitations of a rough interface are the socalled capillary waves. These are interface height correlations whose equilibrium correlation function is of the form 20,21

$$
\left\langle h(\mathbf{k}, 0) h^{*}(\mathbf{k}, 0)\right\rangle \sim \frac{1}{k^{2}} .
$$

(For a finite system of size $L$, the small $k$ divergence is cut off at wave vector of order $L^{-1}$.) As will be discussed in Sec. III, our data also support the validity of Eq. (7) for the PNG model.

Consider now the height correlation function at the same position, $\left\langle[h(\mathbf{r}, t)-h(\mathbf{r}, 0)]^{2}\right\rangle$. With the aid of (5) and (7) we obtain

$$
\begin{aligned}
&\left\langle[h(\mathbf{r}, t)-h(\mathbf{r}, 0)]^{2}\right\rangle \\
& \quad=\frac{1}{2 \pi^{2}} \int d \mathbf{k}\left\langle h(\mathbf{k}, 0) h^{*}(\mathbf{k}, 0)\right\rangle\left(1-e^{-D_{\mathrm{kin}} k^{2} t}\right) \\
& \sim \int_{0}^{k_{\max }} d k k^{-1}\left(1-e^{-D_{\mathrm{kin}} k^{2} t}\right) \\
& \sim \ln t .
\end{aligned}
$$

This result shows that the vertical broadening of the mean-square height fluctuations is much slower than for a diffusion process $\left(\sim t^{1 / 2}\right)$, so that indeed in a light scattering experiment only the dynamics of lateral correlations [of the form (5)] will be detectable.

It is interesting to note that for the PNG model on a one-dimensional interface the integration over a onedimensional wave vector results in a factor $k^{-2}$ rather than $k^{-1}$ in the second line of (8). As a result, the transverse correlations in the one-dimensional PNG model are predicted to behave diffusively ${ }^{22}$ $\left(\left\langle[h(\mathrm{r}, t)-h(\mathrm{r}, 0)]^{2}\right\rangle \sim t^{1 / 2}\right)$. This behavior will be discussed in more detail in Sec. VI.

\section{MONTE CARLO SIMULATION OF THE PNG MODEL}

We have extended the earlier Monte Carlo simulations of the PNG model (Ref. 2) to test Eqs. (5) and (6) and the picture on which their derivation is based. In the simulations, discrete nucleation events are generated at randomly located lateral positions on the surface. The appropriate vertical position for each event is determined by a comparison with previous events to calculate the height of the surface at that point.

We simulate a finite area of linear size $L$ with periodic boundary conditions. To ensure that many clusters nucleate within one layer, proper parameter values have to be chosen. Most of the data were taken with $V=10^{-4} J L^{3}$, so that according to (1)

$$
\frac{L}{\lambda}=10^{4 / 3} \simeq 21.5 \text {. }
$$

Thus, typically about 400 clusters nucleate in each layer. We simulated $4 \times 10^{6}$ nucleation events, corresponding to growth of some $10^{4}$ layers.

The crystal surface is represented in a rectangular coordinate frame with the $z$ axis the normal growth direction. The $(x, y)$ coordinates of the nucleation events are generated as pairs of random numbers in the interval between 0 and $L$. The time between events is calculated with a third random number $Z$ between 0 and 1, using the relation

$$
\Delta t=-\frac{1}{J L^{2}} \ln Z \text {. }
$$

This method produces values of $\Delta t$ that satisfy a Poisson distribution with an average value of

$$
\left(J L^{2}\right)^{-1}=10^{-8 / 3} \tau \simeq 0.0022 \tau .
$$

The nucleation event is recorded in a list that is subdivided according to the layer in which the event is located. The $x$ and $y$ coordinates of each new event are compared with those for previous events to determine whether a cluster from an earlier event has expanded to cover the point. This comparison is performed starting with the clusters at the highest level, and proceeding down to the lower levels. When a cluster is found that satisfies this condition, the new event is assigned to the next level above, and the process is repeated. To reduce the amount of memory needed, only the clusters in the upper fifteen layers are retained in memory. We have found that fifteen layers is enough to ensure that the lowest level is always completely covered before it is removed from the memory.

We introduce dimensionless quantities (indicated by a tilde) that measure lateral lengths in units of $\lambda$ and time in units of $\tau\left[=10^{8 / 3}\left(J L^{2}\right)^{-1}\right.$ in most of our simulations]. Thus we expect on the basis of (6)

$$
D_{\text {kin }}=\widetilde{D}_{\text {kin }} \frac{\lambda^{2}}{\tau},
$$

with the dimensionless diffusion coefficient $\widetilde{D}_{\text {kin }}$ of order unity. The spatial Fourier transform $h(\mathbf{k}, t)$ is in our simulations evaluated by summing $\exp (i \mathbf{k} \cdot \mathbf{r}) h(\mathbf{r}, t)$ on a $20 \times 20$ grid, so that the grid spacing is of the order of the cluster size. In view of the periodic boundary conditions, the $x$ component of the dimensionless wave vector $\widetilde{k}$ assumes the discrete values [cf. Eq. (9)]

$$
\tilde{k}_{x}=2 \pi n \frac{\lambda}{L} \simeq 0.29 n, \quad n=1,2, \ldots
$$


and similarly for $k_{y}$. In Fig. 3 we plot the natural logarithm of $\left\langle h(\mathbf{k}, t) h^{*}(\mathbf{k}, 0)\right\rangle$ from the simulation versus $\widetilde{k}^{2} \widetilde{t}$ for $n=1,2,3,4\left(0.29 \leq \widetilde{k}_{x} \leq 1.17 ; \widetilde{k}_{y}=0\right)$. If fluctuations decay indeed as predicted by Eq. (5), the data should fall on straight lines with slope independent of $\widetilde{k}$. Indeed, as is clear from Fig. 3 the data are consistent with such a dependence with

$$
\widetilde{D}_{\text {kin }}=0.37 \pm 0.03 \text {. }
$$

Note also that the data for $n=4$ correspond to a wavelength of the order of $5 \lambda$; for much shorter wavelength of the order of $\lambda$ (5) will not hold, as the interface structure is strongly correlated on such length scales.

As also discussed in Sec. II, we expect the equal time correlation of the interface to be similar to those of a rough interface, or

$$
\left\langle h(\mathbf{k}, 0) h^{*}(\mathbf{k}, 0)\right\rangle \sim \frac{1}{k^{2}} .
$$

This behavior should be observed for $k$ values in the range $2 \pi / L \ll k \ll 2 \pi / \lambda$, i.e., for wavelengths larger than the typical cluster size but much shorter than the system size, since the periodic boundary conditions will tend to enhance the fluctuations with wavelength of order $L$. In Fig. 4, we plot $1 /\left\langle h(\mathbf{k}, 0) h^{*}(\mathbf{k}, 0)\right\rangle$ of the PNG model versus $k^{2}$. The limited range of $k$ values that we can probe does not allow us to extract the $k$ dependence accurately, in the regime where (13) is expected to hold, but the data of Fig. 4 do tend to be consistent with the anticipated scaling behavior.

In conclusion, the data of the simulations presented in Figs. 3 and 4 support the random-walk picture for the dynamics of interface fluctuation, and the idea that up to a change in length scale the long-wavelength properties of

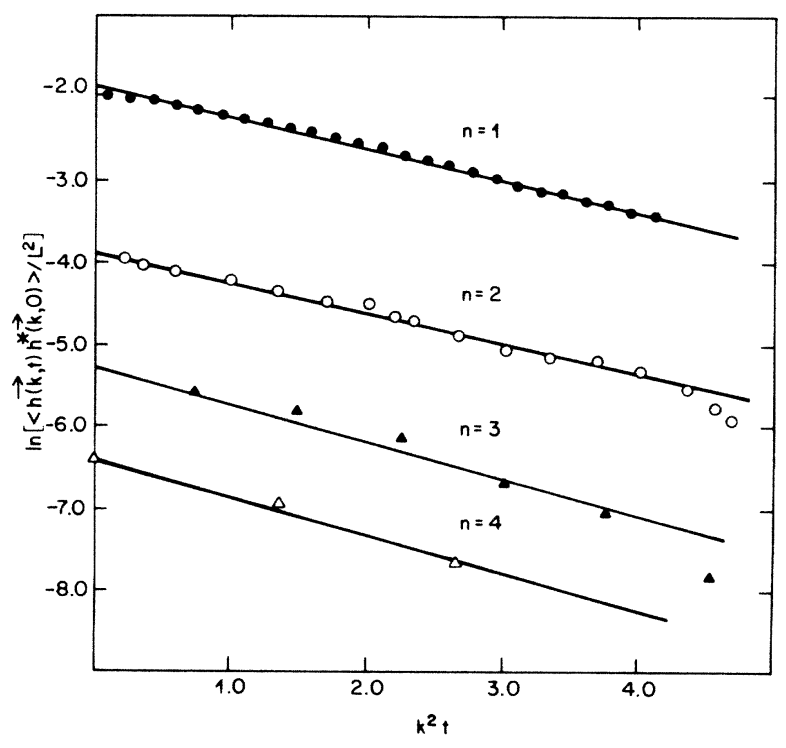

FIG. 3. Data from our Monte Carlo simulations for $\ln \left[\left\langle h(\mathbf{k}, t) h^{*}(\mathbf{k}, 0)\right\rangle / L^{2}\right]$ as a function of the dimensionless combination $\widetilde{k}^{2} \widetilde{t}$. The numbers labeling the curves correspond to the value of $n$ in (11), while $k_{y}=0$. The velocity is $V=10^{-4} J L^{3}$, corresponding to $L=21.5 \lambda$.

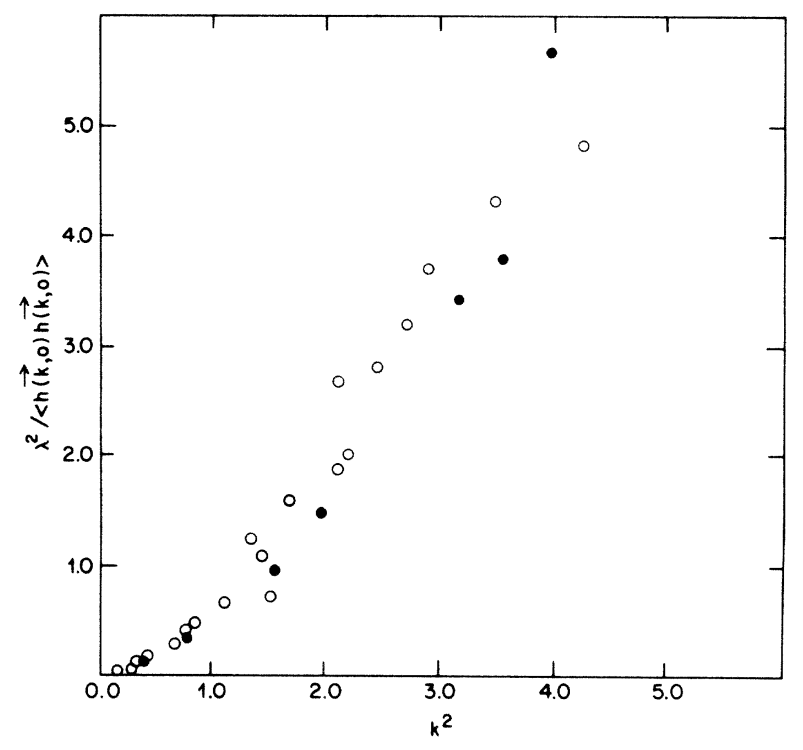

FIG. 4. Inverse of equal time correlation function $\left\langle h(\mathbf{k}, 0), h^{*}(\mathbf{k}, 0)\right\rangle / \lambda^{2}$ of the two-dimensional PNG vs $k^{2}$. The open circles represent data for $V=10^{-4} J L^{3}(L=21.5 \lambda)$ and the closed circles data for $V=10^{-3} J L^{3}(L=10 \lambda)$.

an interface during nucleation controlled growth are like that of a rough interface.

\section{EFFECTS RESULTING FROM THE CURVATURE DEPENDENCE OF THE GROWTH RATE}

Let us now compare the interface dynamics of purely kinematic origin in the PNG model with that of the "corrugated interface model" of Bilgram et al. ${ }^{5,6}$ In this model, the normal growth velocity $V_{n}$ of a crystal is assumed to be a given function of the undercooling $\Delta T$,

$$
V_{n}=\mu(\Delta T)
$$

Let us first consider the effect of small shape fluctuations of a rough interface. In that case, $\Delta T$ is the difference between the melting temperature $T_{m}(\kappa)$ of an interface with curvature $\kappa$ and the interface temperature $T_{i}$. According to the Gibbs-Thomson relation, ${ }^{23,24}$ the former is given by

$$
T_{m}(\kappa)=T_{m}(0)-\frac{\gamma}{\Delta S} \kappa
$$

with $T_{m}(0)$ the melting temperature of a flat interface, $\gamma$ the solid-liquid interface free energy, and $\Delta S$ the entropy of melting. Thus

$$
\Delta T=T_{m}(0)-\frac{\gamma}{\Delta S} \kappa-T_{i}
$$

For small fluctuations $h$ around the steady state $V_{n}^{\mathrm{SS}}=\mu\left(\Delta T^{\mathrm{SS}}\right)$ of a flat interface,

$$
\kappa \simeq-\left(\frac{\partial^{2} h}{\partial x^{2}}+\frac{\partial^{2} h}{\partial y^{2}}\right) .
$$


By linearizing Eqs. (14) and (16) we then get for the dynamics of such fluctuations 5,6

$$
\frac{\partial h}{\partial t}=\mu^{\prime} \frac{\gamma}{\Delta S}\left[\frac{\partial^{2} h}{\partial x^{2}}+\frac{\partial^{2} h}{\partial y^{2}}\right],
$$

where $\mu^{\prime}$ is the derivative of $\mu(\Delta T)$. In deriving this result, we have neglected any fluctuations in the interface temperature $T_{i}$.

Clearly, according to (17) the interface fluctuations in this model behave diffusively with diffusion coefficient $D_{\text {cur }}=\mu^{\prime} \gamma / \Delta S$. However, their dynamics have a different origin than those in the PNG model. In the above derivation, we considered a rough interface, and the diffusive behavior is due to the change of the growth rate with curvature. For positive curvature, i.e., if the solid is bulging forward into the liquid, the growth rate is suppressed. The dynamics of fluctuations in the PNG model, on the other hand, pertain to a faceted interface and are of purely kinematic origin-they exist in the absence of any correlation between the nucleation rate and the interface structure.

The depression of the melting temperature for a curved interface is only properly defined for a rough interface. ${ }^{25}$ Nevertheless, one expects different physical effects to exist during growth of a faceted interface, that have similar consequences. In particular, the nucleation rate could depend on the local structure of the interface: the nucleation close to the clusters might be suppressed (e.g., due to the existence of a depletion layer near a step in vapor growth); similarly, the critical radius for growth of a cluster might have a different inhibition time depending on which level they occupy. Such effects could be modeled by equations of the same form as (14) and (16), but with some unknown coefficient $\epsilon$ instead of $\gamma / \Delta S$ in (16). Although $\gamma / \Delta S$ possibly yields a reasonable estimate for $\epsilon$, we see no reason to expect $\epsilon=\gamma / \Delta S$ for nucleated growth.

In general, therefore, the diffusion coefficient associated with the long-wavelength interface fluctuations of a faceted crystal will be the sum of the two terms $D_{\text {kin }}$ and $D_{\text {cur }}$. Unfortunately, it is difficult to assess which one will dominate in practice. However, for small enough driving force $D_{\text {kin }}$ should dominate, since $D_{\mathrm{kin}} \sim J^{-4 / 3}$ and $D_{\text {cur }} \sim J^{\prime}$, whereas $J \rightarrow 0$ for $\Delta T \rightarrow 0$. Similarly, for increasing driving force $D_{\mathrm{kin}} \sim l^{2} / \tau$ should become less and less important since $l \rightarrow 0$ so that the interface becomes "rough" on shorter and shorter length scales. For an interface that is already rough at equilibrium, $D_{\text {kin }}=0$.

In passing, we note that in an effective interface model, $D_{\text {cur }}$ may actually go to zero near the onset of a MullinsSekerka-type ${ }^{26}$ instability. Such instabilities are most common for growth of a rough interface but analogous effects may exist for growth of a faceted interface. For example, in growth from the vapor the diffusion towards the higher points of the interface is facilitated, and hence the supersaturation and nucleation rate will increase here. (In fact, Frank ${ }^{27}$ has argued that the enhanced supersaturation near corners of a crystal plays a role in the formation of the peculiar shapes of snowflakes.) Such an effect would tend to make $D_{\text {cur }}$ smaller or even negative. It would be interesting to observe such a "critical slowing down."

\section{IMPLICATIONS FOR LIGHT SCATTERING EXPERIMENTS}

Before discussing the possible experimental implications of our results, it is important to point out that in the light scattering experiments on ice, ${ }^{5-7,9,11}$ isotropic scattering was observed with a correlation function different from the oscillatory one mentioned in the introduction. This so-called diffusive scattering is also present in salol: here it was shown ${ }^{11,10}$ to coexist with the oscillatory scattering. The time correlation function of the diffusive scattering decays as $e^{-D k^{2} t}$, where $\mathbf{k}$ is the scattering vector. As yet, no convincing physical interpretation has been given for the "diffusion constant" $D$, which is found to be several orders of magnitude smaller than the thermal diffusivities of water and salol or than a typical solute diffusion constant in the liquid phase of these materials. Bilgram et al. ${ }^{5,6}$ originally associated $D$ with $D_{\text {cur }}$ discussed before. However, in the model of Bilgram et al. the decay rate of fluctuations should be proportional to $k_{\|}^{2}$, with $\mathbf{k}_{\|}$ the component of $\mathbf{k}$ parallel to the interface. When experiments showed that the decay rate is $D k^{2}$ rather than $D k_{\|}^{2}$, this model was rejected. ${ }^{28}$

Fortunately Mesquita et al. ${ }^{11}$ have shown that it is possible to isolate the scattering from interface dynamics from the diffusive scattering by working under conditions of total reflection within the crystal. Under these circumstances there is no light scattered in the melt, and the diffusive scattering is absent, in agreement with the conclusion of Böni et al. ${ }^{9}$ that the diffusive scattering originates on the melt side of the interface. ${ }^{29}$

Our analysis indicates that it would be particularly interesting to do a light scattering experiment on a material during nucleation-dominated growth, with a setup similar to the one of Mesquita et al. ${ }^{11}$ In general, one would expect to see the $e^{-D_{\text {kin }} k^{2} \|^{t}}$ time dependence of interface fluctuation (assuming that $D_{\text {cur }} \ll D_{\text {kin }}$ ). Moreover, if the material is sufficiently anisotropic, it might be possible to measure both the step velocity $V$ and $D_{\text {kin }}$. For, the clusters will become polyganized for large anisotropy, and the steps will all propagate in certain crystallographic directions. Light scattering from these steps would be highly anisotropic and its time correlation function would be damped oscillatory: the damping would result from the finite lifetime $\tau$ of steps and the oscillatory component from the Doppler shift caused by the step motion. The latter measurement would therefore yield $V$. If, in addition, the scattering resulting from the random-walk nature of the interface fluctuations would be detected, one would obtain direct measurements of $D_{\text {kin }}$ and $V$, and therefore of the nucleation rate $R$.

\section{THE ONE-DIMENSIONAL PNG MODEL}

In this section we illustrate the foregoing discussion by explicit calculations for an extension of the onedimensional (1D) PNG model in which the nucleation rate depends on the curvature. In the 1D PNG model, steps nucleate in pairs and then move apart with velocity $\pm V$. As first realized by Frank, ${ }^{1}$ the simplicity of the 1D PNG system lies in the fact that a left moving step with velocity $-V$ is uncorrelated with the right moving steps 
by which it can be annihilated. Let $l(x)$ and $r(x)$ be the densities of steps moving to the left and right. Then the balance equations for these densities read ${ }^{30}$

$$
\begin{aligned}
& \frac{\partial l}{\partial t}=I+V \frac{\partial l}{\partial x}-2 V l r+i, \\
& \frac{\partial r}{\partial t}=I-V \frac{\partial r}{\partial x}-2 V l r+i .
\end{aligned}
$$

Here we have split the total nucleation rate into two terms, a "systematic" part $I$ and a random term $i$ that acts as a random force with zero mean $(\langle i\rangle=0)$. The other terms on the right-hand side of (18) and (19) represent the drift and annihilation of steps. The product form of the annihilation terms reflects the fact mentioned earlier that steps that can annihilate each other are uncorrelated. (This is not true in the 2D model.)

A possible dependence of the nucleation rate on the curvature $\nabla^{2} h$ of the interface is included by writing

$$
I=I_{0}+\frac{1}{2} \epsilon V \nabla^{2} h=I_{0}+\frac{1}{2} \epsilon V \nabla(l-r) .
$$

Here $I_{0}$ is the (position independent) nucleation rate of a flat interface, and $\epsilon$ is a dimensionless parameter; for $\epsilon>0$ the nucleation is suppressed on regions of the interface with positive curvature, as in Bilgram's model. In (20), we also used the fact that the interface length $h$ (measured in units of the layer spacing) is related to $l$ and $r$ by

$$
\nabla h=l-r \text {. }
$$

For convenience, we define $\lambda$ and $\tau$ in this case by

$$
\lambda=\left(\frac{V}{2 I_{0}}\right)^{1 / 2}, \quad \tau=\left(2 I_{0} V\right)^{-1 / 2} .
$$

The average values $\langle l\rangle$ and $\langle r\rangle$ are space and time independent so that using (18)-(22)

$$
\langle r\rangle=\langle l\rangle=\left(\frac{I_{0}}{2 V}\right)^{1 / 2}=\frac{1}{2} \lambda^{-1} .
$$

The average growth rate $R$ is clearly equal to the product of the velocity and the local step density, $R=V(\langle l\rangle+\langle r\rangle)=\lambda^{-1} v=\tau$. Defining in analogy with (3) the average distance $d$ a step travels by $R=2 V d$, we find

$$
d=\lambda .
$$

We are interested in the average relaxation following an initial perturbation in $r$ and $l$ at time $t=0$. Following de Groot and Mazur, ${ }^{31}$ we denote these conditional averages by $\bar{r}$ and $\bar{l}$. Since $\bar{i}=0$ (the random current is uncorrelated with the initial conditions), $\bar{r}$ and $\bar{l}$ obey, according to (18) $-(20)$, the linearized equations

$$
\begin{aligned}
& \frac{\partial \bar{l}}{\partial t}=V\left(1+\frac{1}{2} \epsilon\right) \frac{\partial \bar{l}}{\partial x}-\frac{1}{2} \epsilon V \frac{\partial \bar{r}}{\partial x}-V \lambda^{-1}(\bar{l}+\bar{r}), \\
& \frac{\partial \bar{r}}{\partial t}=-V\left(1+\frac{1}{2} \epsilon\right) \frac{\partial \bar{r}}{\partial x}+\frac{1}{2} \epsilon V \frac{\partial \bar{l}}{\partial x}-V \lambda^{-1}(\bar{l}+\bar{r}) .
\end{aligned}
$$

For modes of the form $e^{-\omega t+i k x}$ we obtain the characteristic equation

$$
\left(\frac{\omega}{V}\right)^{2}-\frac{2}{\lambda}\left(\frac{\omega}{V}\right)+k^{2}(1+\epsilon)=0,
$$

with solutions

$$
\omega_{ \pm}=-\frac{V}{\lambda}\left\{-1 \pm\left[1-k^{2} \lambda^{2}(1+\epsilon)\right]^{1 / 2}\right\} .
$$

In the long-wavelength limit, $\omega_{\text {- }}$ turns out to correspond to fluctuations with $\bar{r} \simeq \bar{l}$ and so, according to (21), $\nabla \bar{h} \simeq 0$. Thus this short-lived long-wavelength mode is mainly connected with local disturbances that do not entail interface height fluctuations; it is of no further interest here.

The long-wavelength $\omega_{+}$mode is indeed related to height fluctuations. For $k^{2} \lambda^{2}(1+\epsilon) \ll 1$, we get

$$
\omega_{+} \simeq \frac{1}{2} V \lambda k^{2}(1+\epsilon)=\frac{1}{2} \frac{d^{2}}{\tau}(1+\epsilon) k^{2},
$$

where $d$ is the average distance a step travels, given by (24). Equation (28) agrees with the discussion of the preceding section in that the effective diffusion coefficient $D$ is the sum of $D_{\text {kin }}$ and $D_{\text {cur }}$, with $D_{\text {kin }}=\frac{1}{2} d^{2} / \tau$, and $D_{\text {cur }}=\frac{1}{2} \epsilon d^{2} / \tau$. Note that $D_{\text {kin }}$ is exactly equal to the diffusion coefficient corresponding to a $1 \mathrm{D}$ random walker that makes steps of size $\pm d$ in every time interval $\tau$. The reason that the picture of Sec. II is also quantitatively correct in this case is a result of the absence of correlations between steps that encounter each other.

In the short-wavelength limit $k>\lambda^{-1}$ and for $\epsilon=0$, $\omega_{+}$, and $\omega_{-}$are damped oscillatory modes of the form

$$
e^{-t / \tau+i k(x \pm V t)}
$$

This form reflects the fact that $l(+\operatorname{sign})$ and $r(-\operatorname{sign})$ consist of steps moving with constant velocity $V$ and whose annihilation gives rise to an average lifetime $\tau$.

These considerations show that the long-wavelength behavior of lateral correlations in the 1D and 2D PNG model have the same origin. The main difference between the two cases is the existence of short length scale correlations in the 2D model which prohibit exact evaluation of $D_{\text {kin }}$.

In Sec. II, we briefly discussed the correlation in time of height fluctuations at the same position, and noted that $\left\langle[h(\mathbf{r}, t)-h(\mathbf{r}, 0)]^{2}\right\rangle \sim t^{1 / 2}$ for large times. This diffusive behavior results if the times at which successive layers cover a position $\mathbf{r}$ occur randomly. Then the times $t_{n}$ at which a position $\mathbf{r}$ is covered in layer $n$ form a Poisson process with mean time $\tau$ between the covering events. For a Poisson process, the probability $P_{n}(t)$ that $n$ covering events have occurred at time $t$ is given by ${ }^{32}$

$$
P_{n}(t)=\frac{(t / \tau)^{n}}{n !} e^{-t / \tau}
$$

Accordingly, if $b$ is the layer thickness, the mean-square height fluctuations are $^{33}$

$$
\left\langle[h(\mathbf{r}, t)-h(\mathbf{r}, 0)]^{2}\right\rangle=b^{2}\left\langle[n(t)-\langle n(t)\rangle]^{2}\right\rangle=b^{2} \frac{t}{\tau} .
$$

This corresponds to a transverse diffusion coefficient 
$D_{\|}=b^{2} / 2 \tau$. Note, however, that although even the transverse correlations are diffusive, there is still a large anisotropy since

$$
\frac{D_{\|}}{D_{\text {kin }}}=\frac{b^{2}}{\lambda^{2}} \ll 1
$$

This ratio is small because in nucleation controlled growth the layer spacing is much smaller than the typical cluster size $\lambda$.

\section{CONCLUSIONS}

In this paper we have discussed the various physical effects that govern the dynamics of interfacial fluctuations during crystal growth by the nucleation and spreading of $2 \mathrm{D}$ clusters. Up to a change in length scale, these fluctuations behave qualitatively the same as those above the roughening transition. That is, in the limit of an infinite surface area the surface height correlations are similar to those in the equilibrium crystal surface above its roughening transition temperature. This is true for arbitrarily small values of the chemical potential driving force for crystal growth, $\Delta \mu$. As $\Delta \mu \rightarrow 0$, the nucleation rate $J$ is expected to decrease much more steeply than the step velocity $V$, and hence the length scale $(V / J)^{1 / 3}$ should diverge. The surfaces of finite crystals will, of course, tend to be localized at a single height during most of the time, except for the period just after a nucleation event while the cluster is expanding to cover the surface. Kinetic roughening occurs only in the sense that the length scale (which measures the average distance between steps on the infinite surface) decreases with increasing $\Delta \mu$; but there is no unique definition of a kinetic roughening transition with an associated value of $\Delta \mu$ at which transition is observed. Only in the case of strict equilibrium is a qualitative change in surface structure observable at a unique transition temperature.

We have shown that the decay of small surface height fluctuations in the 1D PNG model is diffusive in nature, and therefore similar in behavior to the equilibrium surface. Diffusive behavior is also expected for the 2D PNG model, and the Monte Carlo simulations are consistent with this result. The effective diffusion coefficient for this process has the value derived from dimensional arguments, with a dimensionless constant that is approximately unity. Capillarity effects, absent in the PNG model, are shown to increase the effective diffusion coefficient for the decay of the fluctuations.

\section{ACKNOWLEDGMENT}

We are grateful to John D. Weeks for his interest and numerous stimulating discussions.
${ }^{1}$ F. C. Frank, J. Cryst. Growth 22, 233 (1974).

${ }^{2}$ G. H. Gilmer, J. Cryst. Growth 49, 465 (1980).

3J. D. Weeks and G. H. Gilmer, in Advances in Chemical Physics, edited by I. Prigogine and S. A. Rice (Wiley, New York, 1979), Vol. 40.

${ }^{4}$ A. A. Chernov, in Modern Crystallography III, Crystal Growth, edited by A. A. Chernov (Springer, New York, 1984).

5J. H. Bilgram, H. Güttinger, and W. Känzig, Phys. Rev. Lett. 40, 1394 (1978).

${ }^{6}$ H. Güttinger, J. H. Bilgram, and W. Känzig, J. Phys. Chem. Solids 40, 55 (1979).

7J. H. Bilgram, in Nonlinear Phenomena at Phase Transitions and Instabilities, edited by T. Riste (Plenum, New York, 1982), p. 343.

${ }^{8}$ U. Dürig and J. H. Bilgram, Nonlinear Phenomena at Phase Transitions and Instabilities, Ref. 7, p. 371.

${ }^{9}$ P. Böni, J. H. Bilgram, and W. Känzig, Phys. Rev. A 28, 2953 (1983).

10U. Dürig, J. H. Bilgram, and W. Känzig, Phys. Rev. A 30, 946 (1984).

${ }^{11}$ O. N. Mesquita, D. G. Neal, M. Copic, and H. Z. Cummins, Phys. Rev. B 29, 2846 (1984).

${ }^{12}$ R. A. Brown, J. Keizer, U. Steiger, and Y. Yeh, J. Phys. Chem. 87, 4135 (1983).

${ }^{13}$ For a more elaborate discussion see, e.g., Modern Crystallography III, Crystal Growth, Ref. 4, Sec. 3 and D. E. Ovsienko and G. A. Alfintsev, in Crystals: Growth, Properties and Applications, edited by H. C. Freyhardt (Springer, Berlin, 1980), Vol. 2.

${ }^{14}$ See, e.g., V. Bostanov, J. Cryst. Growth 42, 194 (1977), for experiments on electrocrystallization of silver, and G. J. Abbaschian and S. F. Ravitz, J. Cryst. Growth 44, 453 (1978), for measurement of solidification rates of gallium.
${ }^{15}$ Recently there have also been in situ observations of monomolecular steps on growing crystals. See, K. Tsukamoto, J. Cryst. Growth 61, 199 (1983).

${ }^{16}$ Note that since the roughening transition is of infinite order, quantities like the number of broken bonds per interface site appear to vary quite smoothly through the transition. As a result, two interfaces can hardly be distinguishable on a local scale even though one of them is rough and the other one isn't. Consider, e.g., three interfaces, one (A) far below the roughening temperature, the second one $(B)$ somewhat below roughening and the third one $(C)$ just above roughening. Both $A$ and $B$ have a nucleation barrier for growth, but the interface properties (number of broken bonds per site, etc.) of B at microscopic length scales are closer to those of $\mathrm{C}$ than to those of $\mathbf{A}$. Only $\mathbf{C}$ is rough in equilibrium, but all three of them show dynamic roughening.

${ }^{17}$ We refer to Gilmer's paper (Ref. 2) for references to and a discussion of earlier work on the PNG model.

${ }^{18}$ The fact that $c_{1}$ should be of order unity can already be seen from a simple one-layer picture, in which growth takes place in only one layer. The time it takes to form this layer is $1 / R$, and in the typical time $(2 R)^{-1}$ a cluster in this layer grows, it will cover an area $\pi(V / 2 R)^{2}$. Since there are $J / R$ clusters per unit area, one estimates $\pi(V / 2 R)^{2}=R / J$. This yields $R=\frac{1}{2} \pi^{1 / 2} \tau^{-1}$, or $c_{1} \simeq 0.92$. This value is too small mainly because growth in a multilevel structure is more efficient than in a one-level structure. See Ref. 2 for a review of more sophisticated approximations.

${ }^{19}$ This dynamic roughening only occurs if the nucleation rate is high enough so that the typical size of a cluster $\lambda$ is much smaller than the size of a facet. For small nucleation rates, every cluster covers the whole facet.

${ }^{20}$ F. P. Buff, R. A. Lovett, and F. H. Stillinger, Phys. Rev. Lett. 
$15,621(1965)$

21J. D. Weeks, J. Chem. Phys. 67, 3106 (1977).

${ }^{22}$ The transverse height fluctuations for the 1D PNG model resemble those of the random deposition model of J. D. Weeks, G. H. Gilmer, and K. A. Jackson, J. Chem. Phys. 65, 712 (1976). However, if some finite surface tension would be introduced in that model, its behavior on a two-dimensional interface would become more like that of the PNG in two dimensions.

${ }^{23}$ See, e.g., D. Turnbull, in Solid State Physics, edited by H. Ehrenreich, F. Seitz, and D. Turnbull (Academic, New York, 1956), Vol. 3, p. 236.

${ }^{24}$ C. Herring, in Physics of Powder Metallurgy, edited by W. E. Kingston (McGraw-Hill, New York, 1951), p. 157; Structure and Properties of Solid Surfaces, edited by R. Gomer and G. S. Smith (University of Chicago Press, Chicago, 1953), p. 5.

${ }^{25}$ See, in particular, C. Herring, in Physics of Powder Metallurgy, and in Structure and Properties of Solid Surfaces, Ref. 24, for a discussion of this point. In fact, as shown by Herring, terms due to the crystalline anisotropy in $\gamma$ are neglected in (15). Such "surface stiffness" terms actually diverge for a faceted interface.

${ }^{26}$ W. W. Mullins and R. F. Sekerka, J. Appl. Phys. 39, 323 (1963); 35, 444 (1964).

${ }^{27}$ F. C. Frank, Contemp. Phys. 23, 3 (1982).

${ }^{28}$ Other possibilities that have been suggested include the pres- ence of a third phase between the crystal and the melt (Böni et al., Ref. 9), or strong orientational ordering (as in a nematic) near the interface (Mesquita et al., Ref. 11).

${ }^{29}$ In a recent paper, J. Keizer, P. Mazur, and T. Morita [Phys. Rev. A 32, 2944 (1985)] consider it an open question whether the diffusive scattering originates on the melt or crystal side of the interface. They present a defect model, which can only be consistent with scattering originating at the crystal side of the interface.

${ }^{30}$ The normal modes of (18) and (19) with $I$ independent of the curvature are discussed by C. H. Bennett, M. Büttiker, R. Landauer, and H. Thomas, J. Stat. Phys. 24, 419 (1981). See also N. Goldenfeld, J. Phys. A 17, 2807 (1984) for other results on the one-dimensional PNG model.

${ }^{31}$ S. R. de Groot and P. Mazur, Non-Equilibrium Thermodynamics (Dover, New York, 1984), p. 100.

${ }^{32}$ See, e.g., N. G. van Kampen, Stochastic Processes in Physics and Chemistry (North-Holland, Amsterdam, 1981).

${ }^{33}$ We stress that we have only investigated the linearized equations. Recently, M. Kardar, G. Parisi, and Y.-C. Zhang (unpublished) have shown that certain nonlinear terms in the dynamical equations for 1D interface models are relevant. They predict that the 1D mean-square height fluctuations should actually grow as $t^{4 / 3}$ for long times. We thank D. A. Huse for bringing this work to our attention. 